\title{
HÁBITOS DE PUBLICACIÓN DE LA ÉLITE CIENTÍFICA DE ESPAÑA
}

\author{
José Antonio Salvador-Oliván* \\ Departamento de Ciencias de la Documentación e Historia de la Ciencia. Universidad de Zaragoza. \\ Gonzalo Marco-Cuenca** \\ Departamento de Ciencias de la Documentación e Historia de la Ciencia. Universidad de Zaragoza. \\ Rosario Arquero-Avilés ${ }^{* * *}$
}

Facultad de Ciencias de la Documentación. Universidad Complutense de Madrid.

\begin{abstract}
Resumen: El objetivo de este estudio consiste en analizar los patrones de autoría y hábitos de publicación de los investigadores de España más productivos y/o citados.

Método: Se han seleccionado los investigadores españoles o extranjeros que trabajan en instituciones españolas, con mayor índice $\mathrm{H}$ y número de citas recibidas en Google Scholar (GS) a partir del Webometrics Ranking of World Universities. Los datos de las publicaciones se han obtenido de la colección principal de Web of Science (WOS).

Resultados y conclusiones: La productividad e impacto de los investigadores en Ciencias Sociales y Humanidades es mucho menor en la base de datos WOS que en GS. Como autores hiperprolíficos destacan los investigadores en el campo de Física de Partículas, donde es habitual la publicación de 90 artículos al año firmados por más de 2000 autores. Los investigadores de Ciencias de la Salud también son altamente productivos y en multiautoría.

Palabras clave: Multiautoría: autores prolíficos; productividad científica; hábitos de publicación.
\end{abstract}

Title: PUBLISHING HABITS OF SPAIN'S SCIENTIFIC ELITE.

Abstract: This paper aims to analyze the authorship patterns and publication habits of the most productive and / or cited researchers in Spain.

Method: Spanish researchers or those who work in Spanish institutions with the highest $\mathrm{H}$ index and number of citations received in Google Scholar (GS) have been selected from the Webometrics Ranking of World Universities. Publication data was obtained from the main Web of Science (WOS) collection.

Results and conclusions: The productivity and impact of researchers in Social Sciences and Humanities is much less in the WOS database than in GS. As hyperprolific authors highlight researchers in the field of Particle Physics, where the publication of 90 articles per year signed by more than 2000 authors is common. To a lesser extent, Health Sciences researchers are also highly productive in multi-author.

Keywords: Multiple authorship; prolific authors; researcher productivity; publication habits.

Copyright: (C) 2020 Servicio de Publicaciones de la Universidad de Murcia (Spain). Este es un artículo de acceso abierto distribuido bajo los términos de la licencia Creative Commons Reconocimiento 4.0 Internacional (CC BY 4.0).

\section{INTRODUCCIÓN}

La publicación de los resultados de investigaciones, como decía Humphreys (1909) hace más de un siglo, es un deber, convirtiéndose en las últimas décadas en una necesidad y en la principal herramienta que tienen los investigadores para su promoción profesional y obtención de financiación. "Publicar o perecer" es una frase acuñada que describe la presión de los científicos para publicar de manera rápida y continua trabajos académicos.

Esta presión por publicar sigue dominando el mundo académico (Erren, Shaw y Morfeld, 2016, p. 1431) ya que el prestigio y carrera profesional de los investigadores se valora en gran medida por la cantidad de artículos que firman y por el factor de impacto de las revistas donde se publican. Este hecho puede llevar a prácticas poco éticas, entre las que ya se señalaban hace años la de añadir autores supernumerarios (Angell, 1986, p. 261), y a sugerir que un elevado número de publicaciones pudiera incumplir los criterios de autoría aludiendo autoría honoraria o invitada e, incluso, fraude (Wager, Singhvi y Kleinert, 2015).

Es un hecho demostrado y ampliamente documentado en la literatura el aumento del número de autores en las publicaciones de cualquier campo de conocimiento (Wutchy, Jones y Uzzi, 2007; Fortunato et al., 2018; Milojevic, 2014; Adams et al., 2019). En los últimos cinco años, se ha duplicado el número de artículos indizados en Web of

\footnotetext{
*jaso@unizar.es

*** gmarco@unizar.es

*** carquero@ucm.es
}

Recibido: 17-08-2020; $2^{\mathrm{a}}$ versión: 09-10-2020; aceptado: 13-10-2020.

SALVADOR OLIVÁN, J.A.; MARCO CUENCA, G. y ARQUERO AVILÉS, R. Hábitos de publicación de la élite científica de España. Anales de Documentación, 2020, vol. 23, nº 2. Disponible en: http://dx.doi.org/10.6018/analesdoc.440101. 
Science (WOS) con más de 1.000 autores (Singh Chawla, 2019). Esta práctica, denominada hiperautoría, era común en Física de Partículas y Nuclear, pero se ha ido extendiendo a otros campos relacionados con la medicina y cambio climático. En estos artículos, no hay forma de conocer, a partir de las publicaciones, la contribución concreta de cada individuo.

Como principales causas del aumento en el número de autores se han identificado la complejidad cada vez mayor de la investigación y el aumento de la colaboración entre múltiples centros nacionales e internacionales con el consiguiente desarrollo de grandes grupos de investigación. La colaboración en ciencia es necesaria, y la coautoría o autoría compartida su natural consecuencia, presentando entre sus múltiples ventajas la posibilidad de explorar cuestiones complejas desde una perspectiva multidisciplinar que no podrían tratarse de otra manera, o compartir costes (Nabout et al., 2015, p. 362).

El crecimiento en el número de autores ha provocado un debate sobre el significado de autoría, quién está justificado que aparezca como autor y cómo deben reconocerse los distintos tipos de contribuciones en una investigación. En una investigación hay muchos tipos de contribuciones y la autoría no es la única manera que existe para reconocerlas.

Los requisitos más ampliamente establecidos para aparecer como autor son los recomendados por el Comité Internacional de Editores de Revistas Médicas (ICMJE, siglas en inglés), apropiados también en otros campos diferentes de las ciencias biomédicas. En ellos se especifica que la autoría debe basarse en el cumplimiento de los cuatro siguientes criterios (International Committee of Medical Journal Editors (ICMJE), 2020): a) Contribución sustancial a la concepción o diseño del estudio; o adquisición, análisis o interpretación de los datos del trabajo; b) elaboración del borrador del trabajo o revisión crítica del contenido intelectualmente importante; c) aprobación de la versión final que se va a publicar; y d) ser responsable de todos los aspectos del trabajo. Todos aquellos designados como autores deben cumplir los cuatro criterios de autoría; los que incumplan alguno de ellos, deben figurar en las secciones de agradecimientos o reconocimientos. Algunos investigadores consideran estos criterios demasiado estrictos o incluso poco éticos (Shaw, 2011, p. 1).

El European Code of Conduct for Research Integrity, elaborado por más de 50 academias europeas de ciencia de más de 40 países, señala que todos los autores son responsables del contenido de una publicación y que la autoría se basa en una contribución significativa al diseño de la investigación, colección de datos o análisis e interpretación de los resultados (ALLEA - All European Academies, 2017). Pero no aclara el significado de "contribución significativa"; la ambigüedad en el concepto de autoría implica diferentes interpretaciones en distintas disciplinas y países (Larivière et al., 2016; Hosseini et al., 2020).

Springer, una de las principales editoriales de revistas, declara que, aunque no existe una definición universal de lo que constituye la autoría, el grupo de investigación debería identificar a los autores que han contribuido suficientemente al trabajo científico, que son responsables de su parte del trabajo y que revisaron críticamente y aprobaron la versión final del manuscrito. Los criterios a cumplir son: autoría, redacción, revisión y aprobación (Springer, 2013).

En parecidos términos se expresa Raff (2003, p. 57) al indicar que solo deberían aparecer como autores los que han participado en a) el diseño del estudio, b) adquisición de datos, c) análisis e interpretación de datos, y d) elaboración y edición del artículo, y en la sección de reconocimientos aquellos que solo han hecho contribuciones técnicas. Además de otorgar crédito donde se debe, esto protegería a los coautores de cualquier responsabilidad si se descubriera una mala conducta científica en partes de la publicación que no están relacionadas con una contribución específica.

Los editores de revistas han desarrollado sus propias guías sobre qué constituye una autoría válida; sin embargo, no dejan de ser guías, y existen discrepancias entre ellas a pesar de que los editores declaran seguir las pautas establecidas por el ICMJE (Teixeira da Silva y Dobránszki, 2016, p. 1459).

El reconocimiento de autoría implica una contribución de manera más o menos importante a la concepción del estudio y a la elaboración del manuscrito. La hiperautoría ha cambiado lo que constituye la contribución de un autor a una publicación y ha provocado que, en estas situaciones, el concepto de autoría pierda su sentido y se cuestione su valor (Cronin, 2001). Todos aquellos que hacen aportaciones al trabajo deberían ser reconocidos de manera apropiada, aunque no siempre como autores. Solo los que han tenido un papel de liderazgo importante en el proyecto, deberían identificarse como autores junto con el nombre del grupo (Jones, 2011, p. 32). 
Autores que publican más de 72 artículos en un año (lo que equivale a un artículo cada cinco días) se consideran prolíficos inverosímiles, si bien no hay evidencias de que hagan algo inapropiado, sino que es el resultado en muchos casos de que en algunos campos de conocimiento los equipos de investigación han operacionalizado su propio concepto y significado de autoría (Ioannidis, Klavans y Boyack, 2018, p. 167). Aunque algunas de las principales justificaciones de estos autores por su extrema productividad fueron su compromiso con la investigación científica, mucho trabajo y amor por la investigación, Ioannidis, Klavans y Boyack $(2018$, p. 168) argumentan que no son incentivos tan fuertes como para publicar tanto.

El concepto de autores tan prolíficos desafía el concepto de autoría y se ha demostrado que cuando el número de autores es tan elevado, un listado ordenado de autores no es apropiado para fines de atribución y crédito. Las publicaciones en revistas son uno de los principales criterios empleados en la evaluación de científicos y académicos para promoción, acceso a plazas y financiación de proyectos. El problema puesto de manifiesto en la actualidad es que no todos los autores de una publicación contribuyen de la misma manera en una investigación y en la elaboración del manuscrito, pero las comisiones de evaluación pueden otorgar el mismo crédito de autoría a todos los firmantes del artículo. Los autores hiperprolíficos no solo deberían medirse por el número de publicaciones sino también por la utilidad de sus investigaciones medida por el número de citas que reciben (Bornmann y Tekles, 2019, p. 707).

Por ello se ha sugerido, en estos casos, pasar del modelo de autoría al modelo de contribución (Brand et al., 2015; McNutt et al., 2018), similar a los créditos que aparecen al final de una película en forma de una larga lista de personas con sus contribuciones descritas completa y específicamente (Munafo y Smith, 2018, p. 401), que reflejaría mejor la gran variedad de contribuciones que se pueden hacer en los grandes proyectos internacionales.

Comunicar a la revista la contribución individual de cada autor es una opción para conocer qué hizo cada uno, pero en artículos con muchos autores añade longitud y complejidad al listado, aunque solo apareciera electrónicamente. Pero si surgen dudas sobre la validez del trabajo en su conjunto, ya sea como desafíos a su conducta o como evaluaciones de su influencia en el campo, un equipo es un equipo, y sus miembros deben compartir el crédito o la culpa (Kennedy, 2003, p. 733). Tradicionalmente, el primer autor es el que más contribuye, mientras que la posición del resto de autores se decide bien por su contribución, bien por orden alfabético, o por antigüedad en orden inverso. La tradición es diferente según los países y campos de conocimiento, y el significado de la posición se ha hecho cada vez más arbitrario conforme ha aumentado el número de autores, y no hay una forma de determinar el crédito de autoría asociado con el orden de los autores (Tscharntke et al., 2007, p. 13).

Las disciplinas deben adoptar un sistema de crédito académico que recompense una mayor variedad de roles más específicamente, como el sistema CRediT (the Contributor Roles Taxonomy) que permite describir las tareas realizadas y la contribución específica de cada colaborador en un artículo (Holcombre, 2019, p. 147).

En esta línea, muchas revistas permiten, e incluso requieren, a los autores que declaren su contribución en los artículos. Incluso, se han desarrollado taxonomías y vocabularios estandarizados para describir los roles de los autores. Del mismo modo, los comités de promoción y tenencia están utilizando esta información de contribución en sus evaluaciones, adquiriendo una información más completa sobre la contribución de un investigador que la que se obtiene de una lista simple de autores (Kiser, 2018, p. 435).

En un informe del grupo Web of Science de Clarivate Analytics se recomienda que los artículos con hiperautoría (más de 100 autores y/o 30 países) se traten de manera diferente y se excluyan de cualquier análisis realizado a nivel nacional e institucional ya que conducen a tasas de citas muy elevadas. La hiperautoría produce patrones particularmente diferentes y erráticos en la medicina clínica y física de partículas, que no se repiten en todas las disciplinas y no son consistentes (Adams et al., 2019).

En la actualidad, es habitual ver anuncios en periódicos y en las propias universidades que presumen de que determinados académicos son autores de cientos de publicaciones. Este tipo de reconocimiento y los sistemas de recompensa académica que existen en muchos países donde parece que se valora más la cantidad que la calidad de las publicaciones de un investigador, puede ser parte importante del problema (Wager, 2015, p. 2). La productividad de un investigador se mide generalmente por el número de publicaciones. Esto puede ser razonable mientras la productividad, medida a través de simples números, no se convierta en un medio para medir la calidad del personal académico y no se tenga en cuenta también la contribución realizada a los artículos publicados (Langer, 2017, p. 429).

La ciencia está impulsada principalmente por los esfuerzos de una fracción pequeña de investigadores que publican la mayoría de la investigación científica y obtienen la mayoría de las citas (Parker, Allesina y Lortie, 2013, p. 469). A 
nivel de país, se ha hallado una relación directa entre el número de investigadores altamente citados y altamente productivos (Basu, 2006, p. 367).

\section{OBJETIVOS}

El objetivo general de este trabajo consiste en analizar los patrones de autoría de investigadores españoles y extranjeros que trabajan en instituciones españolas que combinan mucha productividad e impacto -la élite científica-, así como distintas métricas de sus publicaciones.

Como objetivos específicos, se trata de conocer:

- La diferencia entre los datos de productividad e impacto de las publicaciones de los investigadores en Google Académico (GS) y en la colección principal de WOS (revistas de mayor impacto en su campo).

- Número de firmas por documento y su relación con la productividad.

- En qué porcentaje de artículos aparecen como primeros autores.

- El grado de autoría hiperprolífica.

\section{MÉTODO}

Estudio observacional transversal descriptivo.

Población: Investigadores españoles y extranjeros que trabajan en el extranjero. Marco muestral: Webometrics Ranking of World Universities (Cybermetrics Lab, 2020). La fecha de descarga del ranking de investigadores se realizó el 19 de abril de 2020.

Criterios de inclusión: Investigadores con alta visibilidad por su productividad e impacto. Para ello, se seleccionaron aquellos que tenían un índice H en GS superior o igual a 100 (99 autores), y los 100 investigadores con mayor número de citas recibidas. La muestra final estuvo formada por 123 autores, ya que solo 24 de los más citados tenían un índice $\mathrm{H}$ menor de 100.

Criterios de exclusión: Se eliminaron cinco investigadores por las siguientes causas:

1. No encontrar su perfil en GS; lo había eliminado.

2. Muchas publicaciones en GS no eran suyas, y en WOS no se encontraron publicaciones. En mayo de 2020, su perfil en GS mostraba 1 cita en total, y seguía con publicaciones que no eran suyas.

3. Muchas de las publicaciones de GS no eran suyas. Solo tenía 7 publicaciones en WOS.

4. Solo tenía tres publicaciones en WOS.

5. Muchas de las publicaciones de GS no eran suyas. Solo tenía 9 publicaciones en WOS y 1 cita.

Una vez eliminados, el número total de investigadores analizados ha sido de 118, de los que 97 tenían un índice $\mathrm{H}$ en GS mayor de 100 y 21 presentaban un índice H menor de 100.

Extracción y curación de datos: Los indicadores se crearon a partir de los datos de la colección principal de Web of Science (WOS). La elección de esta base viene determinada porque contiene la investigación que se publica en las principales revistas internacionales (core journals) y con más impacto en sus respectivas especialidades, siendo los canales de publicación de muchos investigadores altamente citados (Szomszor, Pendlebury y Adams, 2020).

El método para extraer los datos fue el siguiente:

- Se buscó cada investigador en GS y se actualizaron sus datos de índice H y número de citas. Se anotó la especialidad y la institución de trabajo; si no estaban, se buscaron en Google, recogiendo también el nombre completo.

- Se buscó a cada investigador en la Colección Principal de Web of Science, a través de la opción Búsqueda de autores. Dado que dos o más investigadores pueden tener los mismos apellidos e iniciales, y que pueden aparecer registrados de diferentes formas (una inicial, dos iniciales, nombre completo... etc.), se introdujeron diferentes combinaciones de apellidos con nombres completos o iniciales, y se seleccionaron aquellos que tenían la misma especialidad y/o institución de trabajo que la registrada en GS. Se combinaron los resultados.

- Se exportaron todos los registros del autor en formato completo a un fichero en formato csv, y posteriormente a Excel.

- Para cada autor, se verificó en el fichero Excel su especialidad en las columnas de categorías temáticas (WC$\mathrm{SC})$. De aquellas que no coincidían con las especialidades del autor, se comprobó que los artículos contenían 
una firma correcta (coincidían apellidos e iniciales). Se eliminaron aquellos registros que no se correspondían con el autor (aparecían con otras iniciales, aunque con el mismo o mismos apellidos).

- Una vez depurados los errores identificados en los datos extraídos de la colección principal de WOS, se crearon las siguientes variables:

- Número total de autores en un artículo.

- Número de artículos en los que el autor aparecía en primera posición.

- Número total de citas en WOS.

- Número total de documentos publicados en WOS.

- Índice H en WOS a partir del número de documentos y número de citas.

- Número de artículos publicados en cada uno de los últimos 5 años: 2019-2015. En 14 autores se seleccionaron años anteriores consecutivos (2013-2017, 2014-2018, 2012-2016, 2010-2014) ya que en los últimos años no habían publicado o tenían 1 o 2 documentos; solo en tres autores se seleccionaron los años más recientes no consecutivos debido a que en el resto no tenían publicaciones.

Para describir y analizar los resultados, los autores se clasificaron en los siguientes campos de conocimiento según su especialidad (tanto la indicada en GS como en sus páginas web o de las instituciones de trabajo):

- Astronomía/Astrofísica.

- Ciencias de la Naturaleza: incluye Biología, Ecología, Agricultura y Clima.

- Ciencias de la Salud

- Ciencias Sociales: incluye Sociología y Economía.

- Física de Partículas (de alta energía).

- Física: especialistas en otras áreas de Física (Óptica cuántica, Física de la materia condensada, Fotónica).

- Humanidades: Lingüística y Educación.

- Ingeniería: incluye Informática, Bioinformática, Inteligencia artificial.

- Química.

También se clasificaron en hiperprolíficos y prolíficos. Se consideraron hiperprolíficos aquellos que publicaron 60 artículos o más al menos en 1 año, y prolíficos los que publicaron 25 artículos o más al menos en 1 año.

Proceso y análisis de datos: El fichero Excel se exportó al SPSS v.22, con el que se procesaron los datos y se obtuvo la estadística descriptiva (frecuencias absolutas y relativas para variables cualitativas, media y percentiles para variables cuantitativas) e indicadores.

\section{RESULTADOS}

La mayor parte de investigadores analizados pertenecen al área de Ciencias de la Salud y Física de Partículas (Tabla I). Todos ellos tienen un índice H en GS superior a 100 salvo 21 autores, que se distribuyen de la siguiente manera: 1 en Física de Partículas $(\mathrm{H}=85) ; 3$ en Astronomía (menor valor $\mathrm{H}=79$ ); 8 en Ciencias de la Salud (menor valor $\mathrm{H}=73$ ); 4 en Ciencias de la Naturaleza (menor valor $\mathrm{H}=63) ; 1$ en Ingeniería $(\mathrm{H}=67)$; 3 en Ciencias Sociales (varía de 43 a 74); 1 en Humanidades $(\mathrm{H}=65)$. En Física y Química, todos los autores tienen un índice $\mathrm{H}$ superior a 100.

\subsection{Productividad e impacto en GS vs WOS}

Al comparar el índice $\mathrm{H}$ y ${ }^{\circ}$ de citas obtenidos en GS y WOS, se observa que el mayor descenso medio porcentual se produce en los autores de las áreas de Humanidades y de Ciencias Sociales. Las razones pueden ser debidas a que en estos campos son más frecuentes otro tipo de publicaciones no indizadas en WOS (como libros, capítulos de libros, comunicaciones a congresos) y, de la misma manera, un número elevado de citas proceden de libros, actas de congresos y revistas no indizadas en WOS. 


\begin{tabular}{|c|c|c|c|c|c|c|c|c|}
\hline & \multicolumn{2}{|c|}{ Investigadores } & \multicolumn{3}{|c|}{$\begin{array}{l}\text { Promedio Índice H (GS) } \\
-(\text { WOS) y reducción \% }\end{array}$} & \multicolumn{3}{|c|}{$\begin{array}{c}\text { Promedio } \text {. }^{\circ} \text { citas }(\mathrm{GS})-(\mathrm{WOS}) \text { y } \\
\text { reducción } \%\end{array}$} \\
\hline & $\mathrm{n}$ & $\%$ & H-GS & $\mathrm{H}-\mathrm{WOS}$ & $\%$ & GS & WOS & $\%$ \\
\hline Física de Partículas & 33 & $(28,0 \%)$ & 152,0 & 91,6 & $37,67 \%$ & 124.992 & 45.237 & $56,60 \%$ \\
\hline Astronomía/Astrofísica & 6 & $(5,1 \%)$ & 101,8 & 81,8 & $19,32 \%$ & 67.295 & 42.747 & $35,03 \%$ \\
\hline Física & 4 & $(3,4 \%)$ & 117,1 & 98,7 & $15,79 \%$ & 69.464 & 45.150 & $34,86 \%$ \\
\hline Química & 6 & $(5,1 \%)$ & 128,6 & 98,1 & $24,43 \%$ & 76.584 & 49.168 & $36,69 \%$ \\
\hline Ciencias de la Salud & 50 & $(42,4 \%)$ & 119,4 & 88,9 & $25,67 \%$ & 80.155 & 38.608 & $50,99 \%$ \\
\hline Ciencias de la Naturaleza & 10 & $(8,5 \%)$ & 103,9 & 72,6 & $39,71 \%$ & 61.356 & 29.196 & $51,35 \%$ \\
\hline Ingeniería & 4 & $(3,4 \%)$ & 105,7 & 72,0 & $35,97 \%$ & 79.879 & 38.584 & $53,75 \%$ \\
\hline Ciencias Sociales & 3 & $(2,5 \%)$ & 61,0 & 19,3 & $68,01 \%$ & 66.511 & 8.258 & $86,17 \%$ \\
\hline Humanidades & 2 & $(1,7 \%)$ & 100,0 & 21,5 & $76,49 \%$ & 97.450 & 4.311 & $94,89 \%$ \\
\hline Total & 118 & $(100,0 \%)$ & & & & & & \\
\hline
\end{tabular}

Tabla I. Distribución de investigadores por áreas de conocimiento y reducción porcentual media del índice H y número de citas en WOS comparado con los obtenidos en GS.

Los investigadores del campo de Física de Partículas presentan el mayor índice H en GS, siendo superior a 100 en 32 de ellos (97\%), 27 de los cuales es superior a 115 y 3 superior a 200; mientras que en WOS, solo hay 9 autores con un índice $\mathrm{H}$ igual o superior a 100, y ninguno supera el valor de 115.

En Ciencias de la Salud, 42 autores (84\%) tienen un índice H en GS igual o superior a 100, y 2 de ellos superan el valor de 200, mientras que en WOS solo $13(26 \%)$ tienen un índice igual o superior a 100 y ninguno supera el valor de 200 .

La menor reducción en cuanto al número de citas y del índice $\mathrm{H}$ se produce en los campos de Física, Química y Astronomía. Sorprende la reducción más elevada en Física de Partículas al compararla con estos campos afines. Quizá pueda ser debido a que muchas de las citas recibidas por los artículos puedan proceder de preprints, no indizados en WOS, pero sí presentes en repositorios como Arxiv.

\subsection{Número de firmas por documento}

En la Tabla II se muestran diferentes estadísticos descriptivos (media y percentiles) sobre el número de firmas por documento en cada campo. Destaca especialmente Física de Partículas, donde el número medio de firmantes en los artículos es de 1840; la mitad de los investigadores aparecen como autores de artículos con más de 2087 firmas, y un $25 \%$ de ellos presenta un promedio superior a 2298. El número máximo de autores que aparece en una publicación es de 7651. Destaca también el campo de Astronomía y Astrofísica, con un elevado promedio de autores por documento, y siguen de lejos los autores de Ingeniería y Ciencias de la Salud, con un promedio de 15,5 y 13,9 respectivamente, pero en estos últimos un artículo es firmado por 2467 autores. El menor número de firmas por artículo se da en los campos de Ciencias Sociales y Humanidades.

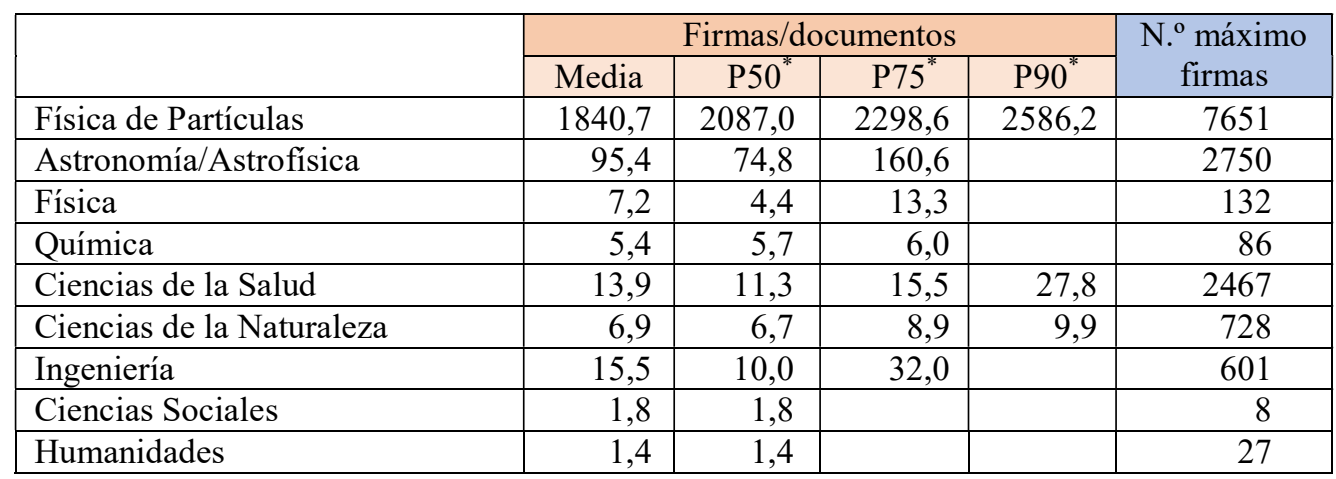

Tabla II. Estadística descriptiva del número de firmas por documento en los investigadores ( ${ }^{\mathrm{P} 50}=$ percentil 50; P75=percentil 50; P90=percentil 90). 
La Tabla III confirma los datos de autoría de la Tabla II. Destaca Física de Partículas, donde casi el $70 \%$ de los artículos están firmados por más de 2.000 autores, y Astronomía/Astrofísica, con más de la 1/4 parte de los artículos firmados por más de 100 autores. En sentido contrario, casi todos los artículos de los campos de Ciencias Sociales y Humanidades están firmados por menos de 6 autores.

\begin{tabular}{|l|r|r|r|r|r|c|}
\hline & \multicolumn{7}{|c|}{ N. ${ }^{\circ}$ firmas/documento } \\
\cline { 2 - 7 } & \multicolumn{1}{|c|}{$<=5$} & \multicolumn{1}{c|}{$6-50$} & $50-100$ & $101-1000$ & $>1000$ & $>2000$ \\
\hline Física de Partículas & $4,2 \%$ & $1,5 \%$ & $0,7 \%$ & $22,9 \%$ & $70,7 \%$ & $69,47 \%$ \\
\hline Astronomía/Astrofísica & $39,7 \%$ & $22,2 \%$ & $10,3 \%$ & $27,1 \%$ & $0,7 \%$ & $0,13 \%$ \\
\hline Física & $73,5 \%$ & $24,1 \%$ & $2,1 \%$ & $0,3 \%$ & & \\
\hline Química & $62,5 \%$ & $37,2 \%$ & $0,3 \%$ & & & \\
\hline Ciencias de la Salud & $30,5 \%$ & $67,4 \%$ & $1,2 \%$ & $0,8 \%$ & $0,05 \%$ & \\
\hline Ciencias de la Naturaleza & $61,6 \%$ & $37,7 \%$ & $0,5 \%$ & $0,2 \%$ & & \\
\hline Ingeniería & $58,6 \%$ & $36,3 \%$ & $2,1 \%$ & $2,8 \%$ & & \\
\hline Ciencias Sociales & $99,2 \%$ & $0,8 \%$ & & & & \\
\hline Humanidades & $99,5 \%$ & $0,5 \%$ & & & & \\
\hline
\end{tabular}

Tabla III. Porcentaje de artículos según número de firmas/documento en los distintos campos.

Existe una relación lineal positiva entre el número de publicaciones y el número de autores, siendo los investigadores que más publican aquellos que firman con muchos autores $(\mathrm{r}=0,480, \mathrm{p}<0,001)$. Al analizar esta relación en los campos que contienen más investigadores, es mucho más intensa en Física de Partículas $(\mathrm{r}=0,895)$ y Ciencias de la Naturaleza $(\mathrm{r}=0,942)$ y algo menos en Ciencias de la Salud $(\mathrm{r}=0,655)$.

\subsection{Firma como primer autor}

El porcentaje de artículos en los que los investigadores aparecen como primer autor es muy bajo en todos los campos, salvo en Humanidades y Ciencias Sociales, donde el número de firmas es también mucho menor (Figura 1). En el campo de Física de Partículas, 6 investigadores no han publicado ningún artículo como primer firmante, y otros 5 solo firman como primer autor en 1 artículo, siendo el porcentaje medio de artículos en los que aparecen como primer autor muy bajo. En Ciencias de la Salud, 1 investigador nunca ha firmado artículos en primer lugar.

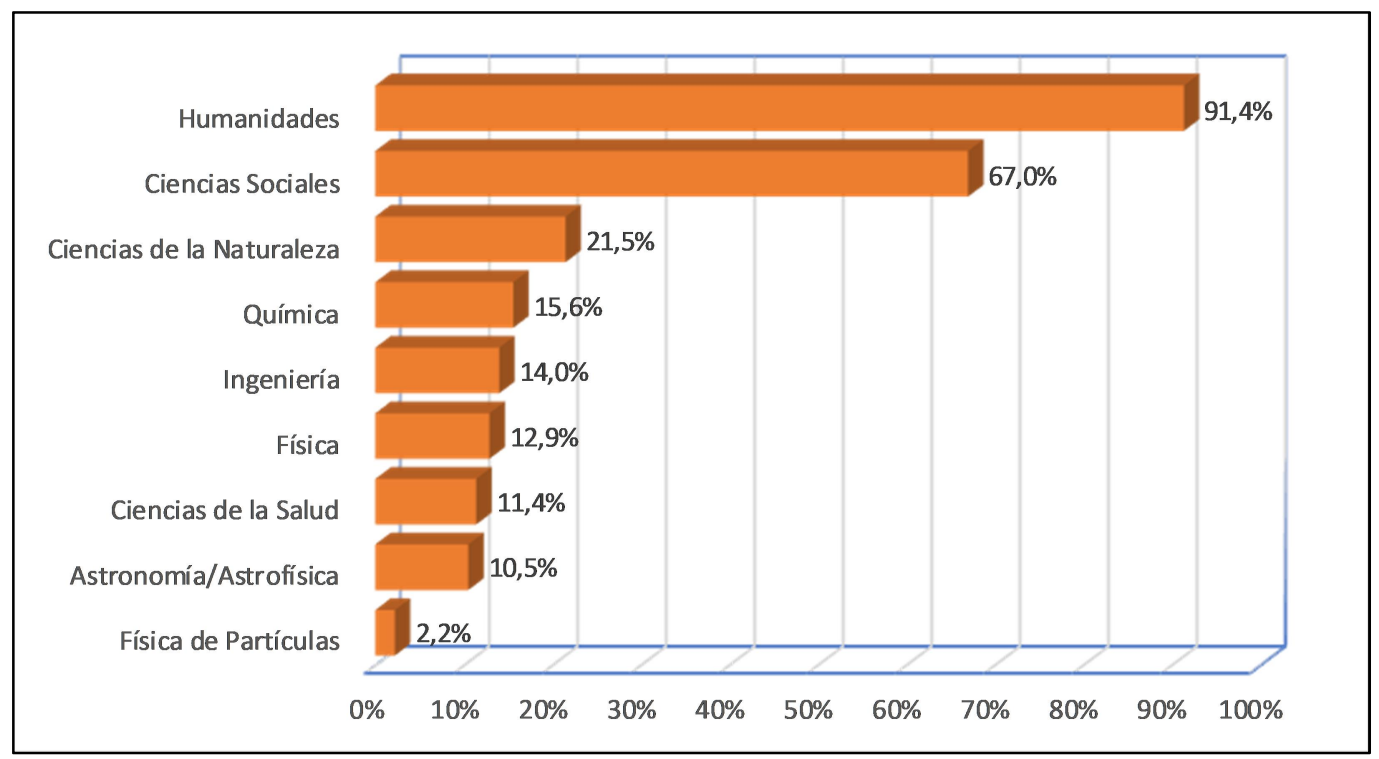

Figura 1. Porcentaje promedio de artículos con el investigador como primer firmante.

\subsection{Hiperproducción}

En la Tabla IV se presenta el número de investigadores en cada campo que han publicado un número elevado de artículos al menos durante 1 año, y durante cuántos años han mantenido esa producción; para ello, se han dividido en aquellos que ha publicado 60 o más artículos (hiperprolíficos) y los que han publicado 25 artículos o más (prolíficos). 


\begin{tabular}{|c|c|c|c|}
\hline & Investigadores hiperprolíficos & Investigadores prolíficos & $\begin{array}{l}\text { N. }{ }^{\circ} \text { docs/ } \\
5 \text { años }\end{array}$ \\
\hline Física de partículas & $\begin{array}{l}29 \text { autores }(87,9 \%) \\
-20 \text { durante } 5 \text { años } \\
-5 \text { durante } 4 \text { años } \\
-1 \text { durante } 3 \text { años } \\
-3 \text { durante } 2 \text { años }\end{array}$ & $\begin{array}{l}31 \text { autores }(93,9 \%) \\
-27 \text { durante } 5 \text { años } \\
-1 \text { durante } 4 \text { años } \\
-2 \text { durante } 3 \text { años } \\
-1 \text { durante } 1 \text { año }\end{array}$ & 89,7 \\
\hline Astronomía/Astrofísica & $\begin{array}{l}1 \text { autor }(16,7 \%) \\
- \text { solo en } 1 \text { año }\end{array}$ & $\begin{array}{l}5 \text { autores }(83,3 \%) \\
-3 \text { durante } 4 \text { años } \\
-2 \text { durante } 3 \text { años }\end{array}$ & 27,4 \\
\hline Física & Ningún autor & $\begin{array}{l}3 \text { autores }(75 \%) \\
-1 \text { durante } 5 \text { años } \\
-1 \text { durante } 3 \text { años } \\
-1 \text { durante } 2 \text { años }\end{array}$ & 22,8 \\
\hline Química & $\begin{array}{l}1 \text { autor }(16,7 \%) \\
-1 \text { durante } 2 \text { años }\end{array}$ & $\begin{array}{l}5 \text { autores }(83,4 \%) \\
-4 \text { durante } 5 \text { años } \\
-1 \text { durante } 1 \text { año } \\
\end{array}$ & 33,5 \\
\hline Ciencias de la salud & $\begin{array}{l}13 \text { autores }(26 \%) \\
-4 \text { durante } 5 \text { años } \\
-1 \text { durante } 4 \text { años } \\
-5 \text { durante } 3 \text { años } \\
-3 \text { durante } 1 \text { año }\end{array}$ & $\begin{array}{l}39 \text { autores }(78 \%) \\
-26 \text { durante } 5 \text { años } \\
-5 \text { durante } 4 \text { años } \\
-2 \text { durante } 3 \text { años } \\
-2 \text { durante } 2 \text { años } \\
-4 \text { durante } 1 \text { año }\end{array}$ & 37,6 \\
\hline $\begin{array}{l}\text { Ciencias de la Natura- } \\
\text { leza }\end{array}$ & $\begin{array}{l}2 \text { autores }(20 \%) \\
-1 \text { durante } 3 \text { años } \\
-1 \text { durante } 1 \text { año }\end{array}$ & $\begin{array}{l}5 \text { autores }(50 \%) \\
-3 \text { durante } 5 \text { años } \\
-1 \text { durante } 3 \text { años } \\
-1 \text { durante } 2 \text { años } \\
\end{array}$ & 23,7 \\
\hline Ingeniería & $\begin{array}{l}1 \text { autor }(25 \%) \\
\quad-1 \text { durante } 1 \text { año }\end{array}$ & $\begin{array}{l}\text { autores }(50 \%) \\
-1 \text { durante } 5 \text { años } \\
-1 \text { durante } 1 \text { año } \\
\end{array}$ & 21,7 \\
\hline Ciencias Sociales & Ningún autor & Ningún autor & 2,1 \\
\hline Humanidades & Ningún autor & Ningún autor & 1,3 \\
\hline
\end{tabular}

Tabla IV. Hiperproducción de documentos publicados en un rango de 5 años.

Destacan principalmente los investigadores del campo de Física de Partículas, siendo 29 los que han publicado 60 artículos o más y, de ellos, 20 han mantenido esa producción durante los últimos 5 años. Destaca también el campo de Ciencias de la salud, donde el 26\% de los investigadores han publicado en un año más de 60 documentos, y 4 han alcanzado estas cifras durante 5 años consecutivos. Salvo en los campos de Ciencias Sociales y Humanidades, en los que ningún autor ha publicado 25 artículos en un año, en el resto hay autores prolíficos o hiperprolíficos. La sobreproducción alcanza unos valores extremadamente altos en el campo de Física de Partículas, con una media de 89,7 artículos publicados durante 5 años consecutivos, seguido de Ciencias de la Salud y de Química con más de 30 artículos.

\section{DISCUSIÓN}

Este trabajo se ha centrado en un conjunto de investigadores, aquellos que tienen un índice $\mathrm{H}$ alto en Google Académico y, por tanto, han publicado un elevado número de artículos y han sido citados muchas veces. Esto significa que este grupo está formado por investigadores principales e influyentes en sus respectivos campos de conocimiento, de los que se han descrito características y hábitos de autoría que pueden ayudar a conocer las razones por las que logran un alto reconocimiento y prestigio tanto a nivel social como académico y forman parte de la élite científica. 


\subsection{Hiperautoría}

En los resultados de nuestro estudio destacan principalmente los autores del campo de Física de Partículas, donde el 70\% de los artículos están firmados por más de 2.000 autores. Unos datos que coinciden con los resultados en otros estudios. Así, Smith (2016, p. 10) ya señalaba que los artículos con más de 100 autores han encontrado un hábitat principalmente en el campo de Física de Alta Energía. Los trabajos con cientos a miles de autores son la norma en una comunidad de miles de científicos que trabajan en proyectos realizados por grandes grupos internacionales formados por más de 1.000 investigadores y donde todos firman como autores como marca identitaria de grupo sin que hayan tenido que escribir o revisar el artículo (Ioannidis, Klavans y Boyack, 2018, p. 168).

La hiperautoría en el campo de Física de Partículas es muy frecuente y fruto de la colaboración, ya que los investigadores comparten instalaciones y observatorios, y forman parte de grandes grupos. En la tabla V se observa que todos los investigadores de Física de Partículas de nuestro estudio participan en grandes grupos, principalmente ATLAS y CMS.

\begin{tabular}{|l|r|r|}
\hline & N. $^{\circ}$ & $\%$ \\
\hline CMS & 12 & $36,4 \%$ \\
\hline ATLAS & 11 & $33,3 \%$ \\
\hline LHCb & 4 & $12,1 \%$ \\
\hline ALICE & 2 & $6,1 \%$ \\
\hline Delphi & 2 & $6,0 \%$ \\
\hline CMS/ATLAS & 1 & $3,0 \%$ \\
\hline IceCube & 1 & $3,0 \%$ \\
\hline
\end{tabular}

Tabla V. Grupos de colaboración de investigadores en Física de Partículas.

ATLAS es un experimento de colisión de partículas del CERN, y utiliza uno de los dos detectores de uso general en el Large Hadron Collider (LHC) en Suiza. En ATLAS participan 3.000 científicos de 172 institutos procedentes de 37 países, y se publican megaartículos. Karl Jakobs, físico y portavoz de colaboración de ATLAS, indica que hay un equipo editorial formado por dos o tres personas que son los que discuten, presentan un bosquejo y escriben el documento, con aportes de los científicos que proporcionaron los datos. Los documentos se someten a una serie de revisiones internas por pares y el equipo editorial recibe los comentarios de los colaboradores, seguido de una revisión institucional externa de un departamento de física colaborador (Leeming, 2019, p. s37). El Compact Muon Solenoid (CMS), junto con ATLAS, es un detector de propósito general diseñado para explorar la física y las colisiones en el LHC.

En grupos tan numerosos, resulta imposible verificar y evaluar la validez de la autoría (Teixeira da Silva y Dobránszki, 2016, p. 1460). Que aparezcan miles de autores en las publicaciones se debe a un reconocimiento generalizado de que ninguna investigación podría hacerse sin las contribuciones de todos los individuos, y se desea reconocer este hecho y motivar la contribución de todos. Todos los físicos participan en el diseño y construcción de sus aceleradores y detectores, y buscan reconocimiento por estas contribuciones de servicio. Por esta razón, todos los participantes en las investigaciones aparecen como autores, ordenados alfabéticamente, de artículos publicados por cualquier miembro, aunque la mayoría admiten que la autoría significa poco cuando hay tantos autores (Birnholtz, 2006, p. 1769). Pero aparecer como autor en tantas publicaciones de este tipo va aumentando la reputación y prestigio de estos investigadores.

En nuestro estudio también es frecuente la multiautoría, aunque con menor número de autores, en otros campos como la Astronomía/Astrofísica, Ciencias de la Salud e Ingeniería. Coincide con otros estudios en los que se ha señalado un elevado número de autores en Física y en Ciencias del Espacio, así como en las áreas de Ciencias de la Salud y Ciencias de la Vida (Adams et al., 2019; Ioannidis, Klavans y Boyack, 2018). En determinadas especialidades médicas es habitual la colaboración y la coautoría donde la condición sine qua non de un proyecto serio financiado es compartir recursos complejos, instalaciones, experiencia, técnicas e individuos (Cronin, 2001, p. 563).

Las normas de justificación de autoría difieren según el campo y varían para cada revista. Hay situaciones en las que se justifica un gran número de autores, como son proyectos internacionales, multicéntricos y multidisciplinares, o el aumento de la complejidad de la investigación, pero también puede ser debido a la presión de "publicar o perecer" (Papatheodorou, Trikalinos y Ioannidis, 2008, p. 551).

El problema no es la hiperautoría en sí, cuando está justificada, sino la existencia de prácticas no éticas como la autoría honorífica o regalada, en las que aparecen como autores individuos que no han participado en la investigación. 
En un estudio realizado en investigadores de la comunidad valenciana, 477 autores $(20,4 \%)$ que habían participado en actividades de colaboración consideraba que las publicaciones científicas no reflejaban de manera adecuada el trabajo realizado en colaboración, siendo el principal motivo para el $43 \%$ de estos autores la existencia de hiperautorías injustificadas, es decir, autores que habían firmado sin haber contribuido a su realización (Gómez-Ferri y González-Alcaide, 2018, p. 9).

\subsection{Firma como primer autor}

Nuestro estudio revela que los investigadores de campos en los que las publicaciones tienen un elevado número de autores, firman pocos artículos como primer autor, y algunos de ellos no lo hacen nunca. Datos que concuerdan con el estudio de Ioannidis, Klavans y Boyack (2018, p. 169), donde el 42,5\% de los autores hiperprolíficos aparecían en las últimas posiciones y solo el 7,1\% aparecían en primera posición. También es similar el resultado en Ciencias de la Naturaleza (21,5\% de artículos como primer autor) con el obtenido por Parker, Allesina y Lortie (2013, p. 475) en la disciplina de Medio Ambiente y Ecología, donde los autores solo aparecían como primer autor en el 26,72\% de los artículos.

\subsection{Hiperproducción científica}

En nuestro estudio sobresale la producción de los investigadores en Física de Partículas, con una publicación media de 90 artículos durante los últimos cinco años, resultados que coinciden con los obtenidos en el estudio de Ioannidis, Klavans y Boyack (2018, p. 167), donde el 86\% de los autores hiperprolífícos procedían del campo de la Física de Partículas y de Alta Energía.

Se ha constatado a lo largo de los años un fuerte aumento en el número de artículos con más de 50 autores, destacando los campos de Biología Molecular y Genética, Neurología e Inmunología con más de 100 autores, y un aumento particularmente notable en aquellos que superan los 1.000 autores, sobre todo en el campo de la Física de Partículas, y especialmente con artículos firmados por más de 3.000 autores derivados del trabajo de la colaboración ATLAS con el Large Hadron Collider (King, 2012).

El número de citas que reciben los investigadores también es mayor en aquellos que pertenecen a campos donde la hiperproducción es mayor. Lógicamente, puede ser debido a que, a mayor número de artículos publicados, mayor probabilidad de que aumente el número de citas. Pero también puede ser debido al efecto Mathew o ventaja acumulativa, que dice que cuanto mayor sea el número de artículos a los que contribuye un autor, más se le conoce y, por lo tanto, es probable que atraiga más citas (Merton, 1968), habiéndose encontrado evidencias de que cuanto mayor es el número de artículos que un investigador publica, mayor es la proporción de estos artículos que están entre los más citados (Larivière y Costas, 2016, p. 1).

Puede ser que los científicos altamente citados hayan acumulado suficiente capital científico a lo largo del tiempo (por ejemplo, acceso a datos, colaboradores expertos, fondos, habilidades técnicas, etc.) para permitirles ser altamente productivos y dedicar menos horas en etapas posteriores de su carrera, beneficiándose así de la ventaja acumulativa (efecto Matthew). (Parker, Lortie y Allesina, 2010, p. 138).

\subsection{Limitaciones}

Los investigadores analizados se han seleccionado de un ranking creado a partir de datos no verificados de Google Académico. Aunque se ha hecho una labor de curación de datos recuperados de la colección principal de WOS, es posible que hayan permanecido algunos pocos registros no propios del autor; esto ha podido provocar una leve variación de los datos en las citas y en el número de publicaciones que no tiene por qué afectar a la visión global de las características presentadas en este estudio.

Otra limitación es que no se refleja la productividad real de cada autor, ya que nuestro análisis se ha limitado solamente a los datos de publicaciones en las principales revistas internacionales, aquellas presentes en el Journal Citation Reports (JCR). No se han incluido publicaciones en otras revistas no indizadas en el JCR y presentes en otras bases de datos como Scopus o en bases de datos que recogen libros. Por lo tanto, la productividad real de cada autor puede estar infraestimada.

También hay que tener presente que investigadores de Humanidades y Ciencias Sociales han podido publicar libros o capítulos de libros no cubiertos en WOS, por lo que los resultados de los investigadores de estas disciplinas seguramente estén menos completos que los obtenidos de disciplinas médicas o de ciencias.

Otra limitación tiene que ver con la posibilidad de identificar de manera adecuada a un autor, condición sine qua non para este tipo de estudios de autores individuales. Las opciones de búsqueda de autores a través de WOS tienen fallos, incluso conociendo el ORCID o su IDResearcher. Se han depurado los datos groseros en los que se ha podido 
identificar claramente que el autor tenía otras iniciales o que publicaban en áreas muy diferentes de su especialidad, pero se han podido "colar" algunos registros en los que pudieran coincidir primer apellido e iniciales con mismas especialidades o afines. Si bien, en estos casos no afectaría al impacto en el índice $\mathrm{H}$ y en el número de publicaciones con un elevado número de citas.

\section{CONCLUSIONES}

En general, hay correspondencia entre los investigadores más productivos y citados a partir de los datos de GS y de la colección principal de WOS, a excepción de cinco casos en los que las publicaciones en GS, y sus citas, no eran suyas. Al comparar la productividad y número de citas recibidas entre GS y WOS, los investigadores de Ciencias Sociales y Humanidades tienen una reducción en el índice $\mathrm{H}$ mucho más elevada que en otros campos de conocimiento.

Los investigadores del campo de Física de Partículas forman parte de grandes grupos de investigación internacionales y publican artículos en los que firman como autores todos los integrantes, siendo lo habitual más de 2.000. En Astronomía/Astrofísica es también muy frecuente la multiautoría, con una media de autores por documento muy próxima a 100. En Ciencias de la Salud, también es frecuente la colaboración y la multiautoría, con una media de 15 firmas por documento. Por el contrario, en Ciencias Sociales y Humanidades, predominan autores únicos o con muy escasa colaboración.

La productividad, medida en número de publicaciones, es mucho mayor en investigadores que participan en grandes grupos de colaboración y con un elevado número de coautores. En estos casos, los investigadores aparecen muy raramente, o nunca, como primer autor.

El número de publicaciones es exagerado en prácticamente todos los investigadores de Física de Partículas, con un promedio de casi 90 artículos por año durante 5 años seguidos (1 artículo cada 4 días), y en la cuarta parte de investigadores en Ciencias de la Salud, con una media de casi 40 artículos por año ( 1 artículo cada 9 días).

La promoción de los investigadores en el mundo académico y profesional, así como el prestigio dado por medios de comunicación, depende, habitualmente, del número e impacto de sus publicaciones. Resulta difícil comprender el significado de autoría en aquellos casos en los que se publica un artículo cada 5 o 10 días, o cada mes, independientemente de la participación en grandes grupos nacionales o internacionales. Las revistas e instituciones deberían pedir a los autores que justificaran su autoría, y de esa manera, dar la importancia que se debe a la integridad y honestidad en la comunicación de los resultados de la investigación, y competir en igualdad de condiciones. Deberían plantearse desafíos éticos que ayuden a la comprensión del significado de autoría, al valor de las publicaciones producidas y a la credibilidad del investigador.

\section{BIBLIOGRAFÍA}

ADAMS, J.; PENDLEBURY, D; POTTER, R. y SZOMSZOR, M. Global Research Report. Multi-authorship and research analytics. Clarivate Analytics, ISI, Web of Science Group, 2019 [en línea]. Disponible en: $<$ https://clarivate.com/webofsciencegroup/campaigns/global-research-report-multi-authorship-and-researchanalysis/> [Consulta: 23 de julio de 2020]

ALLEA - All European Academies. The European code of conduct for research integrity. Berlin: ALLEA, 2017 [en línea]. Disponible en: https://doi.org/10.1142/9789814340984_0003.

ANGELL, M. Publish or perish: A proposal. Annals of Internal Medicine, 1986, vol. 104, no 2, p. 261-262. Disponible en: https://doi.org/10.7326/0003-4819-104-2-261.

BASU, A. Using ISI's "highly cited researchers" to obtain a country level indicator of citation excellence. Scientometrics, 2006, vol. 68, no 3, p. 361-375. Disponible en: https://doi.org/10.1007/s11192-006-0117-x.

BIRNHOLTZ, J. What does it mean to be an author? The intersection of credit, contribution, and collaboration in science. Journal of the American Society for Information Science and Technology, 2006, vol. 57, $\mathrm{n}^{\mathrm{o}}$ 13, p. 17581770. Disponible en: https://doi.org/10.1002/asi.20380.

BORNMANN, L. y TEKLES, A. Productivity does not equal usefulness. Scientometrics, 2019, vol. 118, no 2, p. $705-$ 707. Disponible en: https://doi.org/10.1007/s11192-018-2982-5.

BRAND, A.; ALLEN, L.; ALTMAN, M.; HLAVA, M. y SCOTT, J. Beyond authorship: Attribution, contribution, collaboration, and credit. Learned Publishing, 2015, vol. 28, $\mathrm{n}^{\circ}$ 2, p. 151-155. Disponible en: https://doi.org/10.1087/20150211. 
CRONIN, B. Hyperauthorship: A postmodern perversion or evidence of a structural shift in scholarly communication practices? Journal of the American Society for Information Science and Technology, 2001, vol. 52, n⿳ 7, p. 558569. Disponible en: https://doi.org/10.1002/asi.1097.

CYBERMETRICS LAB. Ranking of Spanish researchers, researchers working in Spanish Institutions (Spain) and Spaniards working abroad according to their Google Scholar Citations public profiles. 15 ed., Consejo superior de Investigaciones Científicas (CSIC), 2020 [en línea]. Disponible en: $<$ http://www.webometrics.info/en/GoogleScholar/Spain $>$ [Consulta: 19 de julio de 2020]

ERREN, T.C; SHAW, D. y MORFELD, P. Analyzing the publish-or-perish paradigm with game theory: The prisoner's dilemma and a possible escape. Science and Engineering Ethics, 2016, vol. 22, no 5, p. $1431-1446$. Disponible en: https://doi.org/10.1007/s11948-015-9701-x.

FORTUNATO, S. et al. Science of science. Science, 2018, vol. 359, n ${ }^{\circ}$ 6379, p. eaao0185. Disponible en: https://doi.org/10.1126/science.aao0185.

GÓMEZ-FERRI, J. y GONZÁLEZ-ALCAIDE, G. Patrones y estrategias en la colaboración científica: La percepción de los investigadores. Revista Española de Documentación Científica, 2018, vol. 41, nº 1, p. 1-17. Disponible en: https://doi.org/10.3989/redc.2018.1.1458.

HOLCOMBRE, A. Farewell authors, hello contributors. Nature, 2019, vol. 571, p. 147. Disponible en: https://doi.org/10.1038/d41586-019-02084-8.

HOSSEINI, M.; CONSOLI, L.; ZWART, H.A.E. y VAN DEN HOVEN, M.A. Suggestions to improve the comprehensibility of current definitions of scientific authorship for international authors. Science and Engineering Ethics, 2020, vol. 26, p. 597-617. Disponible en: https://doi.org/10.1007/s11948-019-00106-2.

HUMPHREYS, W. J. The Duty of publishing. Science, 1909, vol. 30, no 762, p. 177-179.

INTERNATIONAL COMMITTEE OF MEDICAL JOURNAL EDITORS (ICMJE). Defining the role of authors and contributors. 2020 [en línea]. Disponible en: <http://www.icmje.org/recommendations/browse/roles-andresponsibilities/defining-the-role-of-authors-and-contributors.html $>$ [Consulta: 12 de julio de 2020]

IOANNIDIS, J.P.A.; KLAVANS, R. y BOYACK, K.W. Thousands of scientists publish a paper every five days. Nature, 2018, vol. 561, p. 167-169. Disponible en: https://doi.org/10.1038/d41586-018-06185-8.

JONES, D.G. Is multiple authorship in conceptual bioethics ethically sustainable? American Journal of Bioethics, 2011, vol. 11, no 10, p. 30-32. Disponible en: https://doi.org/10.1080/15265161.2011.603809.

KENNEDY, D. Multiple authors, multiple problems. Science, 2003, vol. 301, $\mathrm{n}^{\mathrm{o}}$ 5634, p. 733. Disponible en: https://doi.org/10.1126/science.301.5634.733.

KING, C. Multiauthor papers: onward and upward. Science Watch, 2012 [en línea]. Disponible en: $<$ http://archive.sciencewatch.com/newsletter/2012/201207/multiauthor_papers/> [Consulta: 20 de junio de 2020]

KISER, G.L. No more first authors, no more last authors. Nature, 2018, vol. 561, $\mathrm{n}^{\mathrm{o}}$ 7724, p. 435. Disponible en: https://doi.org/10.1038/d41586-018-06779-2.

LANGER, N. The fundamentals that contribute to the concept of "Publish or Perish". Educational Gerontology, 2017, vol. 43, no 9, p. 429-430. Disponible en: https://doi.org/10.1080/03601277.2017.1332859.

LARIVIÈRE, V. y COSTAS, R. How many is too many? On the relationship between research productivity and impact. PLoS ONE, 2016, vol. 11, no 9, p. 1-10. Disponible en: https://doi.org/10.1371/journal.pone.0162709.

LARIVIÈRE, V.; DESROCHERS, B.M.; MONGEO, P.; PAUL-HUS, A. y SUGIMOTO, C.R. Contributorship and division of labor in knowledge production. Social Studies of Science, 2016, vol. 46, no 3, p. 417-435. Disponible en: https://doi.org/10.1177/0306312716650046.

LEEMING, J. How to manage a multi-author megapaper. Nature, 2019, vol. 575, p. S36-S37. Disponible en: https://doi.org/10.1038/d41586-019-03544-x.

MCNUTT, M.K. et al. Transparency in authors' contributions and responsibilities to promote integrity in scientific publication. Proceedings of the National Academy of Sciences of the United States of America, 2018, vol. 115, $\mathrm{n}^{\circ}$ 11, p. 2557-2560. Disponible en: https://doi.org/10.1073/pnas.1715374115.

MERTON, R.K. The Matthew effect in science. Science, 1968, vol. 159, $\mathrm{n}^{\mathrm{o}}$ 3810, p. 56-63. Disponible en: https://doi.org/10.1126/science.159.3810.56.

MILOJEVIC, S. Principles of scientific research team formation and evolution. Proceedings of the National Academy of Sciences of the United States of America, 2014, vol. 111, $\mathrm{n}^{\circ}$ 11, p. 3984-3989. Disponible en: https://doi.org/10.1073/pnas.1309723111.

MUNAFO, M.R. y SMITH, G.D. Repeating experiments is not enough. Nature, 2018, vol. 553, nº 7689, p. 399-401. Disponible en: https://doi.org/10.1038/d41586-018-01023-3.

NABOUT, J.C. et al. Publish (in a group) or perish (alone): The trend from single- to multi-authorship in biological papers. Scientometrics, 2015, vol. 102, no 1, p. 357-364. Disponible en: https://doi.org/10.1007/s11192-014-13855.

PAPATHEODOROU, S.I.; TRIKALINOS, T.A. y IOANNIDIS, J.P.A. Inflated numbers of authors over time have not been just due to increasing research complexity. Journal of Clinical Epidemiology, 2008, vol. 61, $\mathrm{n}^{\mathrm{o}}$ 6, p. 546551. Disponible en: https://doi.org/10.1016/j.jclinepi.2007.07.017. 
PARKER, J.N.; ALLESINA, S. y LORTIE, C.J. Characterizing a scientific elite (B): Publication and citation patterns of the most highly cited scientists in environmental science and ecology. Scientometrics, 2013, vol. 94, $\mathrm{n}^{\mathrm{o}} 2, \mathrm{p}$. 469-480. Disponible en: https://doi.org/10.1007/s11192-012-0859-6.

PARKER, J.N.; LORTIE, C.J. y ALLESINA, S. Characterizing a scientific elite: The social characteristics of the most highly cited scientists in environmental science and ecology. Scientometrics, 2010, vol. 85, no 1, p. $129-143$. Disponible en: https://doi.org/10.1007/s11192-010-0234-4.

RAFF, H. A suggestion for the multiple author issue. Science, 2003, vol. 302, $\mathrm{n}^{\mathrm{o}}$ 5642, p. 55-57. Disponible en: https://doi.org/10.1126/science.302.5642.55.

SHAW, D. The ICMJE's definition of authorship is illogical and unethical. British Medical Journal, 2011, vol. 343, p. 1-2. Disponible en: https://doi.org/10.1136/bmj.d7192.

SINGH CHAWLA, D. Hyperauthorship: global projects spark surge in thousand-author papers. Nature, 2019 , vol. 576, p. 342. Disponible en: https://doi.org/10.1038/d41586-019-03862-0.

SMITH, G.H. Trends in multiple authorship among papers in astronomy. Publications of the Astronomical Society of the Pacific, 2016, vol. 128, no 970, p. 1-14. Disponible en: https://doi.org/10.1088/1538-3873/128/970/124502.

SPRINGER. Publishing ethics for journals: A guide for editors-in-chief, associate editors, and managing editors. 2013 [en línea]. Disponible en: <https://resource-cms.springernature.com/springer$\mathrm{cms} / \mathrm{rest} / \mathrm{v} 1 /$ content/4252/data/v1> [Consulta: 27 de junio de 2020]

SZOMSZOR, M.; PENDLEBURY, D.A. y ADAMS, J. How much is too much? The difference between research influence and self-citation excess. Scientometrics, 2020, vol. 123, p. 1119-1147. Disponible en: https://doi.org/10.1007/s11192-020-03417-5.

TEIXEIRA DA SILVA, J.A. y DOBRÁNSZKI, J. Multiple authorship in scientific manuscripts: Ethical challenges, ghost and guest/gift authorship, and the cultural/disciplinary perspective. Science and Engineering Ethics, 2016, vol. 22, $\mathrm{n}^{\circ}$ 5, p. 1457-1472. Disponible en: https://doi.org/10.1007/s11948-015-9716-3.

TSCHARNTKE, T.; HOCKBERG, M.E.; RAND, T.A.; RESH, V.H. y KRAUSS, J. Author sequence and credit for contributions in multiauthored publications. PLoS Biology, 2007, vol. 5, $\mathrm{n}^{\mathrm{o}}$ 1, p. 13-14. Disponible en: https://doi.org/10.1371/journal.pbio.0050018.

WAGER, E. Are prolific authors too much of a good thing? British Medical Journal, 2015, vol. 351, p. 1-2. Disponible en: https://doi.org/10.1136/bmj.h2782.

WAGER, E.; SINGHVI, S. y KLEINERT, S. Too much of a good thing? An observational study of prolific authors. PeerJ, 2015, vol. 3, p. e1154. Disponible en: https://doi.org/10.7717/peerj.1154.

WUTCHY, S.; JONES, B.J. y UZZI, B. The increasing dominance of teams in production of knowledge. Science, 2007, vol. 316, p. 1035-1039. Disponible en: https://doi.org/10.1126/science.1136099. 